\title{
A VARIANT OF A THEOREM BY SPRINGER
}

\author{
I. PANIN AND U. REHMANN \\ To the memory of D. K. Faddeev
}

\begin{abstract}
The theorem in question gives a sufficient condition for a quadratic space over a local ring $R$ to contain a hyperbolic plane over $R$.
\end{abstract}

Our main aim in this paper is to prove the following result, which is a variant of the Springer theorem [La] for quadratic spaces over local rings.

Theorem. Let $R$ be a local Noetherian domain that has an infinite residue field of characteristic different from 2. Let $S=R[T] /(F(T))$ be an integral étale extension over $R$. Let $(V, q)$ be a quadratic space over $R$ such that the $S$-quadratic space $\left(V \otimes_{R} S, q \otimes_{R} S\right)$ contains a hyperbolic plane $\mathbb{H}_{S}$. If the degree of the polynomial $F(T)$ is odd, then the space $(V, q)$ already contains a hyperbolic plane over $R$.

This theorem is a main ingredient in the proof of the following result established in $\underline{\mathrm{P}}$.

0.1. Theorem. Let $R$ be a regular local ring, $K$ its field of fractions, and $(V, \varphi)$ a quadratic space over $R$. Suppose $R$ contains a field of characteristic zero. If $(V, \varphi) \otimes_{R} K$ is isotropic over $K$, then $(V, \varphi)$ is isotropic over $R$; i.e., there exists a unimodular vector $v \in V$ with $\varphi(v)=0$.

It is well known that any finite étale extension $S$ of $R$ has the form $S=R[T] /(F(T))$, where $F(T)$ is a monic separable polynomial. If $A$ is a semilocal ring and $(W, \phi)$ is a quadratic space over $A$, then $W$ contains a hyperbolic plane if and only if $W$ contains a unimodular isotropic vector $w$. A vector $w$ is said to be unimodular if $w$ can be taken as the first vector $w_{1}$ of a free $A$-base $w_{1}, \ldots, w_{n}$ of the $A$-module $W$.

\section{§1. Preliminaries}

In this section we formulate two results to be used in the proof of the main theorem. These two results will be proved in 93 , We need to fix some notation:

$k$ is an infinite field $(\operatorname{char}(k) \neq 2)$;

$f(t)$ is a monic separable polynomial of degree $n$ over $k$;

$l=k[t] /(f(t))$ is a separable $k$-algebra;

$\theta=t \bmod f(t)$ is an element of $l$;

$(W, \phi)$ is a quadratic space over $k$ of rank $\geq 3$;

$\left(W_{l}, \phi_{l}\right)$ is the quadratic space $\left(W \otimes_{k} l, \phi \otimes_{k} l\right)$ over $l$;

$W[t]^{(m)}=W \cdot 1 \oplus W \cdot t \oplus \cdots \oplus W \cdot t^{m-1} \subset W[t]=W \otimes_{k} k[t] ;$

$k[t]^{(m)}=k \cdot 1 \oplus k \cdot t \oplus \cdots \oplus k \cdot t^{m-1} \subset k[t]$

2000 Mathematics Subject Classification. Primary 53A04; Secondary 52A40, 52A10.

Key words and phrases. Quadratic forms, Springer's theorem, local domain.

This work is a part of the project SFB-701 at Fakultät für Mathematik, Universität Bielefeld. The first author is also supported by the Presidium of RAS Program "Fundamental Research", an RFBRgrant, and the INTAS-05-1000008-8118 grant. 
$e v: W[t]^{(n)} \rightarrow W_{l}$ is the map given by

$$
e v\left(v_{0}+v_{1} t+\cdots+v_{n-1} t^{n-1}\right)=v_{0}+v_{1} \theta+\cdots+v_{n-1} \theta^{n-1}
$$

$v(\theta) \stackrel{\text { def }}{=} e v(v(t))$ for any $v(t) \in W[t]^{(n)}$;

$\phi^{(n)}: W[t]^{(n)} \rightarrow k[t]^{(2 n-1)}$ is the map given by

$\phi^{(n)}\left(v_{0}+v_{1} t+\cdots+v_{n-1} t^{n-1}\right)=\phi\left(v_{0}\right)+2\left(v_{0}, v_{1}\right) \cdot t+\phi\left(v_{1}\right) \cdot t^{2}+\cdots+\phi\left(v_{n-1}\right) \cdot t^{2 n-2}$.

1.1. Proposition. Suppose the quadratic space $\left(W_{l}, \phi_{l}\right)$ contains a hyperbolic plane $\mathbb{H}_{l}$ as a direct summand. Then there exists a unimodular isotropic vector $w \in W_{l}$, and for any such vector $w$ there exists an element $v(t) \in W[t]^{(n)}$ satisfying the following conditions:

(1) $\phi^{(n)}(v(t)) \in k[t]^{(2 n-1)}$ is a polynomial of degree $2 n-2$;

(2) $\phi^{(n)}(v(t))$ is a separable polynomial;

(3) $\langle w, v(\theta)\rangle \in l^{\times}$, where $l^{\times} \subset l$ is the group of units of $l$;

(4) $\phi_{l}(v(\theta))=0$ in $l$.

1.2. Let $A$ be a semilocal ring, let $\bar{A}=A / \operatorname{Rad}(A)$, and let $(U, \psi)$ be a quadratic space over $A$. Suppose $(U, \psi)$ contains a hyperbolic plane $\mathbb{H}_{A}$ as a direct summand. We shall use "bar" for reduction modulo the $\operatorname{radical} \operatorname{Rad}(A)$. For example, $(\bar{U}, \bar{\psi})$ is the quadratic space $(U / \operatorname{Rad}(A) \cdot U, \bar{\psi})$ over $\bar{A}$.

1.3. Lemma. Let $u \in U$ be a unimodular isotropic vector. Then for any isotropic vector $\mathrm{v} \in \bar{U}$ with $\langle\mathrm{v}, \bar{u}\rangle \in \bar{A}^{\times}$there exists a vector $v \in U$ such that

(1) $\bar{v}=\mathrm{v}$ in $\bar{U}$, and

(2) $\psi(v)=0$.

Proof. Let $v_{0}$ be any vector in $U$ with $\bar{v}_{0}=\mathrm{v}$. Then

$$
v=\frac{-\left\langle v_{0}, v_{0}\right\rangle}{2\left\langle v_{0}, u\right\rangle} \cdot u+v_{0}
$$

is the required isotropic vector. Indeed, $\psi(v)=-\left\langle v_{0}, v_{0}\right\rangle+\left\langle v_{0}, v_{0}\right\rangle=0$ and $\bar{v}=\bar{v}_{0}$, because $\left\langle\bar{v}_{0}, \bar{v}_{0}\right\rangle=\langle\mathrm{v}, \mathrm{v}\rangle=0$. Since $\bar{v}_{0}=\mathrm{v}$, we have $\bar{v}=\mathrm{v}$. The lemma is proved.

1.4. Lemma. Let $k[t]$ be the polynomial ring over the field $k$, and let $(W, \phi)$ be the quadratic space over $k$. Let $w(t)=w_{0}+w_{1} \cdot t+\cdots+w_{n-1} \cdot t^{n-1}$ be an element of $W[t]$ with $w_{i} \in W$. Suppose that the polynomial $\phi^{(n)}(w(t)) \in k[t]$ is separable, and let $g(t) \in k[t]$ be an irreducible polynomial dividing $\phi^{(n)}(w(t))$. Then $w(t)$ does not vanish modulo $g(t)$.

Proof. If $w(t)$ vanishes modulo $g(t)$, then $w(t)=g(t) \cdot u(t)$ for an element $u(t) \in W[t]$. In this case we have

$$
\phi^{(n)}(w(t))=\phi^{(n)}(g(t) \cdot u(t))=g(t)^{2} \cdot \phi^{(n)}(u(t)) \in k[t] .
$$

This relation contradicts the separability of $\phi^{(n)}(w(t))$. Thus, $w(t)$ does not vanish modulo $g(t)$. The lemma is proved.

1.5. Lemma. Let $A$ be a semilocal ring, and let $(U, \psi)$ be a quadratic space over $A$. Let $v \in U$ be a unimodular isotropic vector. Then a hyperbolic plane can be split out of $(U, \psi)$, i.e.,

$$
(U, \psi) \cong\left(U^{\prime}, \psi^{\prime}\right) \perp \mathbb{H}
$$

Proof. This is easy. 


\section{$\S 2$. The PROOF OF THE MAIN THEOREM}

2.1. Theorem. Let $R$ be a local Noetherian domain that has an infinite residue field of characteristic different from 2. Let $S=R[T] /(F(T))$ be an integral étale extension over $R$. Let $(V, q)$ be a quadratic space over $R$ such that the $S$-quadratic space $\left(V \otimes_{R} S, q \otimes_{R} S\right)$ contains a hyperbolic plane $\mathbb{H}_{S}$. If $\operatorname{deg} F(T)$ is odd, then the space $(V, q)$ contains a hyperbolic plane $\mathbb{H}_{R}$.

Proof. The case of $\operatorname{rank}(q)=2$ is obvious. So we may assume rank $(q) \geq 3$. Let $\mathfrak{m}$ be the maximal ideal of $R$, and let $k$ be the residue field $R / \mathfrak{m}$ of $R$. Let $l=S / \mathfrak{m} S=k[t] /(f(t))$, where $f(t)=F[T] \bmod \mathfrak{m}$. Since $S$ is étale over $R$, so is the $k$-algebra $l$. In particular, the $k$-algebra $l$ is separable.

We shall write $\left(V_{R}, q_{R}\right)$ for $(V, q)$ and $(\bar{V}, \bar{q})$ for the reduction modulo $\mathfrak{m}$ of the $R$ quadratic space $\left(V_{R}, q_{R}\right)$. Let $\left(V_{S}, q_{S}\right)$ be the scalar extension of $\left(V_{R}, q_{R}\right)$ up to $S$, and let $\left(\bar{V}_{l}, \bar{q}_{l}\right)$ be the reduction modulo $\mathfrak{m} S$ of the $S$-quadratic space $\left(V_{S}, q_{S}\right)$. Clearly, $\left(\bar{V}_{l}, \bar{q}_{l}\right)=(\bar{V}, \bar{q}) \otimes_{k} l$. Now, set $(W, \phi)=(\bar{V}, \bar{q})$. Then $\left(W \otimes_{k} l, \phi \otimes_{k} l\right)=\left(\bar{V}_{l}, \bar{q}_{l}\right)$, and we shall write $\left(W_{l}, \phi_{l}\right)$ for $\left(\bar{V}_{l}, \bar{q}_{l}\right)$.

By assumption, the space $\left(W_{l}, \phi_{l}\right)$ contains a hyperbolic plane $\mathbb{H}_{l}$ as a direct summand. Thus, we can apply Proposition 1.1. So, using the notation of \$1 we can find a vector $w \in W_{l}$ (unimodular and isotropic) and an element $v(t)=v_{0} \cdot 1+v_{1} \cdot t+\cdots+v_{n-1} \cdot t^{n-1} \in$ $W[t]^{(n)}$ satisfying the following conditions:

(1) $\phi^{(n)}(v(t)) \in k[t]$ has degree $2 n-2$;

(2) $\phi^{(n)}(v(t))$ is a separable polynomial over $k$;

(3) $\langle w, v(\theta)\rangle \in l^{\times}$is an invertible element of $l$;

(4) $\phi_{l}(v(\theta))=0 \in l$, where $\theta=t \bmod f(t)$ is the element of $l$.

Recall that the quadratic space $\left(W_{l}, \phi_{l}\right)$ is the reduction modulo $\mathfrak{m} S$ of the quadratic space $\left(V_{S}, q_{S}\right)$. By Lemma 1.3, the element $v=v(\theta) \in W_{l}$ can be lifted up to a unimodular isotropic vector $\mathrm{v} \in V_{S}$.

Since $S=R[T] /(F(T))$, we have $V_{S}=V_{R} \otimes_{R} R[T] /(F(T))$. Set $\Theta=T \bmod F(T)$. There are elements $\mathrm{v}_{0}, \mathrm{v}_{1}, \ldots, \mathrm{v}_{n-1} \in V_{R}$ such that $\mathrm{v}=\mathrm{v}_{0} \cdot 1+\mathrm{v}_{1} \cdot \Theta+\cdots+\mathrm{v}_{n-1} \cdot \Theta^{n-1}$. Consider the element $\mathrm{v}(T)=\mathrm{v}_{0} \cdot 1+\mathrm{v}_{1} \cdot T+\cdots+\mathrm{v}_{n-1} \cdot T^{n-1}$ in $V_{R} \otimes_{R} R[T]$, and consider the diagram

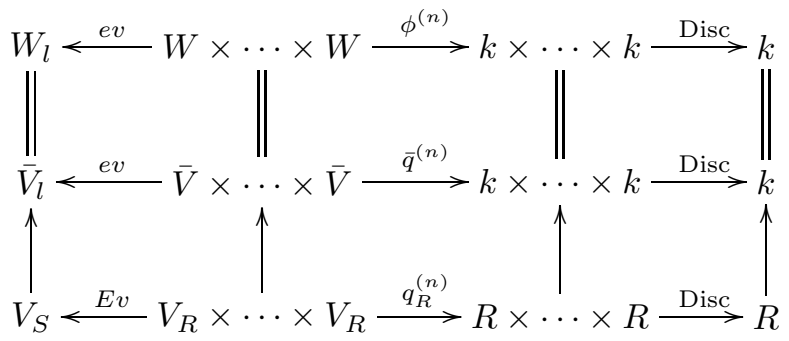

where $E v\left(u_{0}, \ldots, u_{n-1}\right)=u_{0} \cdot 1+u_{1} \cdot \Theta+\cdots+u_{n-1} \cdot \Theta^{n-1} \in V_{S}$,

$$
q_{R}^{(n)}\left(u_{0}, \ldots, u_{n-1}\right)=q_{R}\left(u_{0}\right)+2 \cdot\left(u_{0}, u_{1}\right) \cdot T+q_{R}\left(u_{1}\right) \cdot T^{2}+\cdots+q_{R}\left(u_{n}\right) \cdot T^{2 n-2},
$$

and $\operatorname{Disc}\left(a_{0}, a_{1}, \ldots, a_{2 n-2}\right)$ denotes the discriminant of the polynomial $a_{0}+a_{1} T+\cdots+$ $a_{2 n-2} T^{2 n-2}$. The vertical arrows are canonical maps.

Clearly, this diagram commutes, and the maps $E v$, ev are isomorphisms. In particular, $v_{i}=\mathrm{v}_{i} \bmod \mathfrak{m}$ for the components $v_{0}, \ldots, v_{n-1}$ (respectively, $\mathrm{v}_{0}, \ldots, \mathrm{v}_{n-1}$ ) of the element $v(t) \in W[t]$ (respectively, $\mathrm{v}(T) \in V_{R}[T]$ ). Thus, the reduction modulo $\mathfrak{m}$ of the polynomial $q_{R}^{(n)}(\mathrm{v}(T))$ coincides with the polynomial $\phi^{(n)}(v(t))$. The latter polynomial is separable and has degree $2 n-2$ by the choice of $v(t)$. 
Since $\mathrm{v}(\Theta)=\mathrm{v}$ by the very choice of the element $\mathrm{v}(T)$, and since $\mathrm{v}$ is $q_{S}$-isotropic, we have $q_{R}(\mathrm{v}(\Theta))=0$. Thus, $q_{R}^{(n)}(\mathrm{v}(T))$ vanishes modulo $F(T)$ in the ring $R[T]$; i.e., there exists a polynomial $H(T)$ in $R[T]$ such that

$$
q_{R}^{(n)}(\mathrm{v}(T))=F(T) \cdot H(T) .
$$

After reduction modulo $\mathfrak{m}$, we get the relation

$$
\phi^{(n)}(v(t))=f(t) \cdot h(t),
$$

where $h(t)$ is the reduction of $H(t)$ modulo $\mathfrak{m}$. Since $\operatorname{deg} \phi^{(n)}(v(t))=2 n-2$ and $\operatorname{deg} f(t)=n$, we see that $\operatorname{deg} h(t)=n-2$. Since $\operatorname{deg} H(T) \leq n-2$, we conclude that $\operatorname{deg} H(T)=n-2$ and $H(T)$ is monic (the highest coefficient is invertible). Next, $\phi^{(n)}(v(t))$ is separable. Thus, $h(t)$ is also separable. This shows that the $R$-algebra $S^{\prime}=R[T] /(H(T))$ is an étale $R$-algebra.

Let $A$ denote the class of $T$ modulo $H(T)$ in the ring $S^{\prime}$. Then the vector $\mathrm{v}(A)=$ $\mathrm{v}_{0} \cdot 1+\mathrm{v}_{1} \cdot A+\cdots+\mathrm{v}_{n-1} \cdot A^{n-1}$ of the quadratic $S^{\prime}$-space $\left(V_{S^{\prime}}, q_{S^{\prime}}\right)$ is isotropic. In fact, $q_{S^{\prime}}(\mathrm{v}(A))=F(A) \cdot H(A)=0$ in $S^{\prime}$. If the vector $\mathrm{v}(A)$ is unimodular, then a hyperbolic plane $\mathbb{H}_{S^{\prime}}$ can be split out of the quadratic space $\left(V_{S^{\prime}}, q_{S^{\prime}}\right)$ over $S^{\prime}$ (see Lemma 1.5). So, in this case we have constructed a finite étale extension $S^{\prime}=R[T] /(H(T))$ such that

$$
\operatorname{deg} H(T)=\operatorname{deg} F(T)-2
$$

and the space $\left(V_{S^{\prime}}, q_{S^{\prime}}\right)$ contains a hyperbolic plane $\mathbb{H}_{S^{\prime}}$ as a direct summand. Repeating this procedure several times, finally we get a direct hyperbolic summand of the quadratic space $\left(V_{R}, q_{R}\right)$ itself. Thus, to complete the proof of the theorem it remains to check that the vector $\mathrm{v}(A) \in V_{S^{\prime}}$ is unimodular.

For this, we denote the ring $S^{\prime} / \mathfrak{m} S^{\prime}$ by $k^{\prime}$ and observe that $k^{\prime}=k[t] /(h(t))$, where, as above, $h(t)$ is the reduction modulo $\mathfrak{m}$ of the polynomial $H(t)$. We denote by $\bar{V}_{k^{\prime}}$ the $k^{\prime}$-module $\bar{V} \otimes_{k} k^{\prime}$ and consider the commutative diagram (with the same elements $\mathrm{v}(T)$ and $v(t)$ as above in this proof)

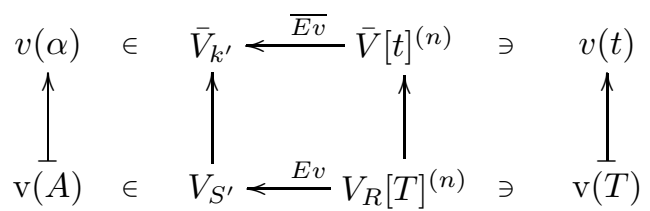

Here $E v\left(u_{0}+u_{1} T+\cdots+u_{n-1} T^{n-1}\right)=u_{0}+u_{1} \cdot A+\cdots+u_{n-1} \cdot A^{n-1}$ and $\overline{E v}\left(w_{0}+\right.$ $\left.w_{1} \cdot t+\cdots+w_{n-1} \cdot t^{n-1}\right)=w_{0}+w_{1} \cdot \alpha+\cdots+w_{n-1} \cdot \alpha^{n-1}$ and $\alpha=t \bmod h(t) \in k^{\prime}$. To check that $\mathrm{v}(A)$ is unimodular, it suffices to verify that $v(\alpha)$ is unimodular. Observe that $\bar{V}_{k^{\prime}}=\bar{V} \otimes_{k} k[t] /(h(t))$. Let $h(t)=h_{1}(t) \cdots h_{r}(t)$ be the factorization of $h(t)$ into irreducible polynomials. Since $h(t)$ is separable, we have $h_{i}(t) \neq h_{j}(t)$ for $i \neq j$. Then $\bar{V}_{k^{\prime}}=\prod_{i=1}^{r} \bar{V} \otimes_{k}\left(k[t] /\left(h_{i}(t)\right)\right)$, so that $v(\alpha)$ is unimodular in $\bar{V}_{k^{\prime}}$ if and only if the elements $v(t) \in \bar{V}[t]$ do not vanish modulo any of the $h_{i}(t)(i=1, \ldots, r)$.

The polynomial $h_{i}(t)$ divides $h(t)$, and the polynomial $h(t)$ divides $\phi^{(n)}(v(t))$ (see $(*)$ ). Therefore, $h_{i}(t)$ divides $\phi^{(n)}(v(t))$. Since $h_{i}(t)$ is irreducible and $\phi^{(n)}(v(t))$ is separable, Lemma 1.4 shows that $v(t)$ does not vanish modulo $h_{i}(t)$. Thus, the element $v(\alpha)$ is indeed a unimodular vector in $\bar{V}_{k^{\prime}}$, and the element $\mathrm{v}(A)$ is a unimodular vector in $V_{S^{\prime}}$. This completes the proof of the theorem.

\section{§3. Proof of Proposition 1.1}

3.1. Since the quadratic space $\left(W_{l}, \phi_{l}\right)$ contains a hyperbolic plane $\mathbb{H}_{l}$ as a direct summand, we can find a unimodular isotropic vector $w \in W_{l}$. We choose and fix such a vector. 
Now, set $X(l)=\left\{v \in W_{l} \mid \phi(v)=0\right\}$ and consider the map $\rho_{w}: X(l) \rightarrow l$ taking $v$ to the scalar product $\langle w, v\rangle \in l$. Then, consider the following diagram of sets and their polynomial maps:

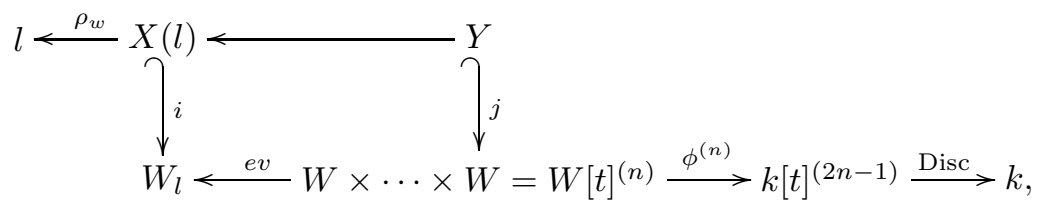

where $e v$ is the map defined at the beginning of $₫ 1$ (clearly, this map is an isomorphism), and where $Y=e v^{-1}(X(l))$, the maps $i, j$ are inclusions, and $\phi^{(n)}$ is defined at the beginning of $\$ 1$ The map Disc takes a polynomial $g(t) \in k[t]^{(2 n-1)}$ to its discriminant. It is well known that $\operatorname{Disc}(g(t))$ has a polynomial expression in terms of the coefficients of $g(t)$.

This diagram is the diagram of $k$-rational points and their maps induced by the following diagram:

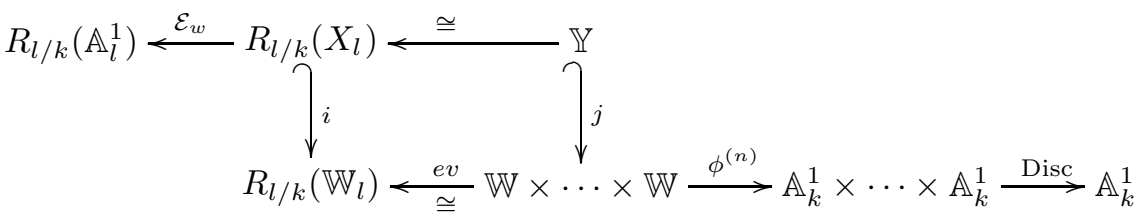

of algebraic varieties. Here $R_{l / k}$ is the Weil restriction functor. Next, $\mathbb{W}$ (respectively, $\mathbb{W}_{l}$ ) is the $k$-vector space (respectively, the $l$-vector space) viewed as a $k$-variety (respectively, as an $l$-variety), $X_{l}$ is the affine quadric in $\mathbb{W}_{l}$ given by the equation $\phi_{l}=0, \mathcal{E}_{w}$ is the Weil restriction of the morphism $\rho_{w}: X_{l} \rightarrow \mathbb{A}_{l}^{1}$ that takes $v$ to $\langle w, v\rangle$, the variety $\mathbb{Y}$ is the preimage of $R_{l / k}\left(X_{l}\right)$ under the evaluation isomorphism $e v: \mathbb{W} \times \cdots \times \mathbb{W} \rightarrow R_{l / k}\left(\mathbb{W}_{l}\right)$, and the morphisms $i$ and $j$ are closed embeddings. The product $\mathbb{W} \times \cdots \times \mathbb{W}$ consists of $n$ factors and the product $\mathbb{A}_{k}^{1} \times \cdots \times \mathbb{A}_{k}^{1}$ consists of $2 n-1$ factors.

We define open subsets of the variety $\mathbb{Y}$ as follows:

$U_{1}=j^{-1}\left(\left(\phi^{(n)}\right)^{-1}\left(\mathbb{A}_{k}^{1} \times \cdots \times \mathbb{A}_{k}^{1} \times\left(\mathbb{A}_{k}^{1}-\{0\}\right)\right)\right)$,

$U_{2}=j^{-1}\left(\left(\phi^{(n)}\right)^{-1}\left(\operatorname{Disc}^{-1}\left(\mathbb{A}_{k}^{1}-\{0\}\right)\right)\right)$,

$U_{3}=e v^{-1}\left(\mathcal{E}_{w}^{-1}\left(R_{l / k}\left(\mathbb{A}_{l}^{1}-\{0\}_{l}\right)\right)\right)$.

We need the following lemmas.

3.2. Lemma. $\mathbb{Y}$ is a $k$-rational variety.

3.3. Lemma. $U_{1} \cap U_{2} \neq \varnothing$.

3.4. Lemma. $U_{3} \neq \varnothing$.

With these three lemmas at hand, we can prove Proposition 1.1 as follows. Since $\mathbb{Y}$ is a $k$-rational variety, any one of its nonempty open subsets has a $k$-rational point (even infinitely many points). Thus, by Lemmas 3.3 and 3.4 , we can find a point

$$
v \in\left(U_{1} \cap U_{2} \cap U_{3}\right)(k) \subset \mathbb{Y}(k) \subset(\mathbb{W} \times \cdots \times \mathbb{W})(k) .
$$

Set $v(t)=j(v)$. Then the element $v(t)$ satisfies conditions (1), (2), and (3) of Proposition 1.1 by the very definition of the open sets $U_{1}, U_{2}$, and $U_{3}$. Since $v(\theta) \stackrel{\text { def }}{=} e v(v(t))$, we see that $v(\theta)$ is a $k$-rational point of the variety $R_{l / k}\left(X_{l}\right)$, i.e., $v(\theta) \in X(l)$. Thus, $\phi_{l}(v(\theta))=0$, and condition (4) is also satisfied. To complete the proof of Proposition 1.1] it remains to prove Lemmas 3.2 3.4 
3.5. Proof of Lemma 3.2. Since $\mathbb{Y}$ is isomorphic to the variety $R_{l / k}\left(X_{l}\right)$, it suffices to check the $k$-rationality of the latter. The quadric $X_{l}$ has an $l$-rational point, so that $X_{l}$ is an $l$-rational variety. This implies the existence of nonempty open subvarieties $V_{1}$ in $X_{l}$ and $V_{2}$ in $\mathbb{A}_{l}^{r-1}\left(r=\operatorname{dim}_{k} W\right)$ and an isomorphism $\alpha: V_{1} \stackrel{\cong}{\longrightarrow} V_{2}$ of $l$-varieties. Consider the diagram of $l$-varieties

$$
X_{l} \supset V_{1} \stackrel{\cong}{\longrightarrow} V_{2} \subset \mathbb{A}_{l}^{r-1}
$$

and apply the Weil restriction functor to this diagram. This leads to a diagram

$$
R_{l / k}\left(X_{l}\right) \supset R_{l / k}\left(V_{1}\right) \stackrel{\cong}{\rightrightarrows} R_{l / k}\left(V_{2}\right) \subset R_{l / k}\left(\mathbb{A}_{l}^{r-1}\right)
$$

of $k$-varieties. Since the left-hand and the right-hand side inclusions are open embeddings and since the variety $R_{l / k}\left(\mathbb{A}_{l}^{r-1}\right)$ is an affine space over $k$, we conclude that $R_{l / k}\left(X_{l}\right)$ is $k$-rational. The lemma is proved.

3.6. Proof of Lemma 3.4. The morphism $e v: \mathbb{Y} \rightarrow R_{l / k}\left(X_{l}\right)$ is an isomorphism by the very definition of the variety $\mathbb{Y}$. Therefore, it suffices to check that the variety

$$
\mathcal{E}_{w}^{-1}\left(R_{l / k}\left(\mathbb{A}_{l}^{1}-\{0\}_{l}\right)\right)
$$

is nonempty. We show that this variety has $k$-rational points. For this, we recall that the morphism

$$
\mathcal{E}_{w}: R_{l / k}\left(X_{l}\right) \rightarrow R_{l / k}\left(\mathbb{A}_{l}^{1}\right)
$$

induces a map of $k$-rational points, which coincides with

$$
\rho_{w}: X(l) \rightarrow l \quad(v \mapsto\langle v, w\rangle) .
$$

Take any $v \in X(l)$ with $\langle w, v\rangle \in l^{\times}$and observe that $\rho_{w}(v) \in l^{\times}$for this element $v$ and moreover, the group $l^{\times}$coincides with the set of $k$-rational points of the variety $R_{l / k}\left(\mathbb{A}_{l}^{1}-\{0\}_{l}\right)$. Thus, the element $v$ is a $k$-rational point of the variety $\mathcal{E}_{w}^{-1}\left(R_{l / k}\left(\mathbb{A}_{l}^{1}-\right.\right.$ $\left.\{0\}_{l}\right)$ ). The lemma is proved.

3.7. Proof of Lemma 3.3. We show that already $\left(U_{1} \cap U_{2}\right)(l) \neq \varnothing$. For this, we consider an $l$-basis $e_{1}, e_{2}, \ldots, e_{r}$ of the free $l$-module $W_{l}$ such that $e_{1}^{2}=0, e_{2}^{2}=0$, $\left(e_{1}, e_{2}\right)=1$, and $\left(e_{1}, e_{i}\right)=\left(e_{2}, e_{i}\right)=0$ for $i \geq 3$. For any polynomial $h(t) \in l[t]^{(n)}$ and any element $e \in W_{l}$, we set

$$
h(t) \cdot e=\left(a_{0} \cdot e\right) \cdot 1+\left(a_{1} \cdot e\right) \cdot t+\cdots+\left(a_{n-1} \cdot e\right) \cdot t^{n-1} \in l \otimes_{k} W[t]^{(n)}=W_{l}[t]^{(n)} .
$$

Since $l=k[t] /(f(t))$ and $\theta=t \bmod f(t)$ (see $\S 1$ for the definition of $\theta$ ), we have a unique decomposition

$$
f(t)=f^{(1)}(t)(t-\theta) \text { in } l[t] .
$$

Let $g(t) \in k[t]$ be a separable polynomial of degree $n-2$ that is coprime to $f(t)$. Consider the element

$$
v(t)=f^{(1)}(t) \cdot e_{1}+(t-\theta) g(t) \cdot e_{2} \in l \otimes_{k} W[t]^{(n)}=W_{l}[t]^{(n)} .
$$

3.8. Claim. $v(t) \in\left(U_{1} \cap U_{2}\right)(l)$.

To check this, first we observe that

$$
\phi^{(n)}(v(t))=2 \cdot f^{(1)}(t) \cdot(t-\theta) \cdot g(t) \cdot\left\langle e_{1}, e_{2}\right\rangle=2 f(t) \cdot g(t) .
$$

Thus,

$$
\phi(v(\theta))=2 \cdot f(\theta) \cdot g(\theta)=0
$$

in $l$, and therefore $v(t) \in \mathbb{Y}(l)$.

Next, $(*)$ shows that $\phi^{(n)}(v(t)) \in k[t] \subset l[t]$ and it has degree $2 n-2$. So, $v(t) \in U_{1}(l)$.

Finally, $\phi^{(n)}(v(t))$ is separable, because $f(t)$ and $g(t)$ are separable and coprime polynomials in $k[t]$. Thus, $\operatorname{Disc}\left(\phi^{(n)}(v(t))\right) \in k^{\times} \hookrightarrow l^{\times}$, whence $v(t) \in U_{2}(l)$. So, $\left(U_{1} \cap U_{2}\right)(l) \ni v(t)$, and consequently, $\left(U_{1} \cap U_{2}\right)(l) \neq \varnothing$. The lemma is proved. 


\section{REFERENCES}

[La] T. Y. Lam, The algebraic theory of quadratic forms, Math. Lecture Note Ser., W. A. Benjamin, Inc., Reading, MA, 1973. MR0396410 (53:277)

[P] I. Panin, Rationally isotropic quadratic spaces are locally isotropic, www.math.uiuc.edu/K-theory/ 0671/2003

St. Petersburg Branch, Steklov Mathematical Institute, Russian Academy of Sciences, Fontanka 27, St. Petersburg 191023, Russia

E-mail address: panin@pdmi.ras.ru

Current address: SFB-701 at Fakultät für Mathematik, Universität Bielefeld, Germany

E-mail address: panin@math.uni-bielefeld.de

Fakultät für Mathematik, Universität Bielefeld, Postfach 100131, D-33501 Bielefeld, GERMANY

E-mail address: rehmann@math.uni-bielefeld.de

Received 30/JUL/2007

Originally published in English 